\title{
Automatically Finding Optimal Working Projections for the Endovascular Coiling of Intracranical Aneurysms
}

\author{
Dale Wilson $^{1}$, J. Alison Noble ${ }^{1}$, Duncan Royston ${ }^{2}$, and James Byrne ${ }^{2}$ \\ 1 Dept. of Engineering Science, University of Oxford, Oxford, OX1 3PJ \\ \{dale, noble\}@robots.ox.ac.uk \\ 2 Dept. of Neuroradiology, Radcliffe Infirmary, Oxford OX2 6HE
}

\begin{abstract}
The endovascular coil embolisation of intracranial saccular aneurysms requires a set of specific $X$-ray images with which to view the aneurysm during coiling. These two-dimensional images, known as working projections should be optimal for measuring the aneurysm sac diameter, inserting the first coil, and checking coil overhang into the surrounding vessels. At present the gantry angle that produces these images is found by the radiologist by trial and error. In this paper we present a method for automatically finding the angles that will produce the desired $X$-ray projections. Our method consists of four steps: (1) segmenting the vasculature from three-dimensional angiographic data; (2) locating the aneurysm neck; (3) labelling the aneurysm sac; and (4) determining the optimal angle for viewing the aneurysm during coiling. We discuss details of the algorithn steps and present the results of the algorithm applied to one synthetic and two pathological examples.
\end{abstract}

\section{Introduction}

A novel technique for the endovascular treatment of intracranial aneurysms with electrolytically detachable platinum coils (Guglielmi detachable coils, GDCs) was described in 1991 [1]. Since then endovascular occlusion of aneurysms with GDCs has become a widely practised technique. Total occlusion of the aneurysm sac can be achieved in up to $90 \%$ of small $(<10 \mathrm{~mm})$ and large $(10-25 \mathrm{~mm})$ aneurysms, but in only $50 \%$ of giant aneurysms $(>25 \mathrm{~mm})$ [2]. Mid-term clinical outcomes are encouraging, with an excellent or good outcome reported in $86 \%$ of patients where the GDC method was the definitive treatment of the aneurysm [3].

Aneurysm coiling is carried out in an X-ray angiography suite where catheters and coils are positioned using two-dimensional $\mathrm{X}$-ray fluoroscopy, road-mapping and digital subtraction angiography (DSA). A clear view of the aneurysm sac and neck and of the adjacent vessels in the image is required to accurately place the coils. However, it may be difficult from visual assessment to determine the best $X$-ray images to use, and even if the optimal projections are found it may still be hard to define the relationship of the aneurysm to the adjacent vessels with $2 \mathrm{D}$ projection information alone.

There are standard projections that are used for the diagnosis of intracranial aneurysms, but these projections can only be a guide to the radiologist choosing the best coiling projection and will not necessarily be optimal. The gantry angles that produce the best projections are generally found using a combination of information from DSA images acquired immediately prior to coiling, the clinical experience of the radiologist, and trial and error. It is not uncommon for up to seven DSA images to be acquired before the radiologist is satisfied with the working projection.

In this paper we propose a protocol for automatically predicting the optimal projections, known as working projections, using 3D angiographic data acquired prior to treatment, and a simulated geometric set-up of an X-ray machine.

The automatic technique presented in this paper is aimed specifically at coiling procedures. However, it is a technique that may well be applied to other areas, such as radiation 
therapy planning. If, for example, a tumour is identified, the optimal gantry angles could be determined based on the geometry of the tumour and surrounding tissues.

The authors know of only one group [4] who have previously worked on a GDC angle prediction method. Building on thejr method we have added several new features: firstly, once our input data has been selected, our algorithm is fully automatic; we treat different aneurysm sites specifically; we include the application specific $\mathrm{X}$-ray machine geometry to improve algorithm accuracy; and finally, we incorporate a sac labelling step into our algorithm, which allows for sac volume estimation.

There are four main steps to our algorithm: (1) segmenting the relevant vasculature from 3D (volumetric) angiography data; (2) determining the location and orientation of the aneurysm neck; (3) labelling all voxels in the segmentation that belong to the aneurysm sac; and (4) combining the information found in the previous steps to predict the angle that produces the best working projection. In this paper we discuss each step of the prediction method, concluding with a discussion of the completed algorithm applied to one synthetically modified data set and two pathological data sets. Details of a study on a larger number of data sets are forthcoming [5].

\section{Segmenting the $3 D$ angiographic data}

Currently there are two clinically available techniques for producing 3D angiographic data: computed tomography angiography and magnetic resonance angiography (MRA). A segmentation of the cerebral vasculature from either data source is suitable input for our viewing angle optimisation algorithm. We use a 3D segmentation from time-of-flight MRA data, because of availability. Details of an early version of the segmentation algorithm are presented elsewhere [6]. The segmentation algorithm must output a 3D binary volume consisting of the arteries that would appear in a DSA image where contrast agent was injected to allow visibility of the aneurysm. Hence the segmentation must be of either the left or right carotid circulation, or the vertebro-basilar circulation, depending on the aneurysm site.

\section{Determining the neck location and orientation}

For our angle prediction algorithm we need to know the neck location to aid in the labelling of the sac, and the neck orientation to initialise the search for the optimal working projection angle. We obtain the geometry of the neck (location and orientation) by analysing spherical projection images computed from voxels inside the parent artery and the aneurysm neck and sac. In the following sections we describe how to choose these voxels and create the spherical projection images, and how these images are used to find the neck position and orientation.

\subsection{Setting up the spline and its local axes To create a spherical projection image we need} a point in 3D and a set of orthogonal coordinate axes originating at that point to parameterise the vectors about the sphere. To define the points we skeletonise a 3D segmentation of the vessels using Euclidean Homotopic Thinning [7] and then fit an approximating open cubic B-spline [8] to the section of the skeleton that passes from the parent artery into the sac. The definition of the coordinate axes is then fairly straightforward, as the spline has intrinsically defined local coordinate axes, the tangent and two normals, at every point along its length. We adjust the two normal vectors so that they vary continuously along the curve, not flipping at inflection points, so that the spherical projection images also vary continuously. It is possible to create spherical projection images at each point on the skeleton if, say, we define each spherical projection image with respect to a global set of $3 \mathrm{D}$ axes. However, this would result 
in the images having no direct relationship to the local geometry of the surrounding vessels, which is a feature we use later.

The spline gives a very good approximation to the medially situated skeleton, with spline points lying within a voxel's distance of the skeleton. In Fig. 1(a) we show a 3D spline fitted to a skeleton, and in Fig. 1(b) show the spline as it is located inside the corresponding segmentation of a posterior communicating artery aneurysm.

3.2 Creating spherical projection images Each pixel position in a spherical projection image references a direction $(\phi, \theta)$ in spherical coordinates that is defined with respect to the local coordinate frame centred at a given point along the spline. We can think of the parameter $\phi$ as longitude and $\theta$ as latitude, where $\phi \in[0,360)$ and $\theta \in[-90,90)$. The two parameters represent the respective indices of the $x$ and $y$ axes of the spherical projection images. The unit vector in 3D Cartesian coordinates with longitude $\phi$ and latitude $\theta$ is $\mathbf{u}(\phi, \theta)=(\cos \theta \sin \phi, \cos \theta \cos \phi, \sin \theta)^{T}$.

The intensity of a pixel in a spherical projection image represents the Euclidean distance from the spline point to the vessel lumen in the direction $\mathbf{u}(\phi, \theta)$. Thus, low intensities in the image indicate directions where the lumen is close to the spline, and higher intensities indicate directions where there is a greater distance between the spline and lumen.

Two typical spherical projection images, one from inside the internal carotid artery, and one from inside a posterior communicating artery aneurysm, are shown in Figs 1(c) and (d) respectively. Note that Fig. 1(c) has high intensity regions at the top and bottom of the images. These regions correspond to $\theta$ close to $-90^{\circ}$ and $90^{\circ}$, where the vectors point up and down the internal carotid artery, and hence there is a large distance to the lumen. In Fig. 1(d) there is only one high intensity region at the bottom of the image, which corresponds to the vectors pointing out through the aneurysm neck. This region will be smaller for small diameter necks, and larger for large necks. Here the neck is quite small.

(a)

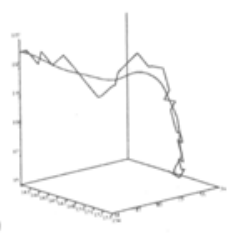

(b)

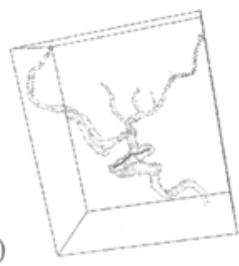

(c)
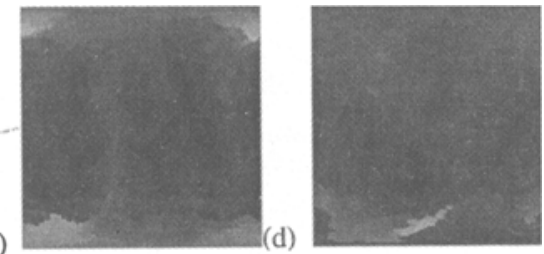

Fig. 1. In (a) a cubic B-spline fitted to a selected part of the segmentation skeleton is shown. In (b) the spline and (a transparent) segmentation of a posterior communicating artery aneurysm are displayed. Two spherical projection images are shown in $(c)$ and $(d):(c)$ is from inside the internal carotid artery, and $(d)$ is from inside the sac of a posterior communicating artery aneurysm.

3.3 Defining the neck parameters Given a sequence of spherical projection images acquired at equal steps along a spline, we want to use them to find the point on the spline that passes through the neck, and the angle of the plane that slices through the least area of the neck. We term this plane the neck plane, and use it later to initialise the optimisation of the working projection angle.

In spherical coordinates, if a plane intersecting the origin is defined by its normal $\left(\phi_{n}, \theta_{n}\right)^{T}$, then all the vectors through the origin that lie parallel to the plane are defined by $\theta=\theta^{\prime} \cos \phi^{\prime}$ 
for all $\phi \in[0,360)$, where $\theta^{\prime}=\theta_{n}+90$, and

$$
\phi^{\prime}= \begin{cases}\phi-\phi_{n} & \text { if } \phi \geq \phi_{n}, \\ (\phi+360)-\phi_{n} & \text { otherwise. }\end{cases}
$$

As $\phi$ varies from 0 to 360 , the $(\phi, \theta)$ pairs around the plane describe points along a sinusoid in a spherical projection image. It is one of these curves that corresponds to the neck plane.

The area of a region in a plane can be approximated by polygonal integration. Referring to the illustration in Fig. 2(a), the area within the triangle pqr is defined as $\frac{1}{2} a . b . \sin (\varphi)$. The distances $a$ and $b$ correspond to intensity values at adjacent positions along one of the sinusoids within a spherical projection image. The area of a plane within the vessel is the sum of all the triangular regions around the plane. To find the plane most likely to be the neck plane, we seek the minimal area over all images and all plane normals $\left(\phi_{n}, \theta_{n}\right)$.

Minimising over the area is not accurate enough to define the neck location and orientation. We must add two normalised weighting functions, one, $\omega^{m}$, over the set of images corresponding to spline points along the skeleton section, and the other, $\omega^{t}$, over the plane normal latitude parameter $\theta_{n}$. These two sets of weights incorporate our prior knowledge of the aneurysm neck in our search for a minimum.
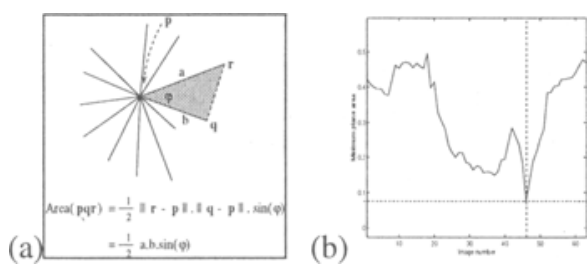

Fig. 2. (a) An illustration of the vectors circumscribing a region of a plane. The definition of the area of one of the triangular regions is also shown. (b) A plot of the weighted minimum plane areas for each image in the sequence for a posterior communicating aneurysm.

The weightings $\omega^{m}$ for the images (and hence spline points) are included because there is no guarantee that the neck diameter will be smaller than the parent artery diameter, so it is possible that the minimum plane area will be found somewhere along the artery before the neck. We avoid this by weighting the images closer to the sac end of the spline with greater likelihood. ${ }^{1}$

The weightings $\omega^{t}$ on the plane normal parameter $\theta_{n}$ simply give greater likelihood to planes with normals closer to the spline tangents. This is because the spline is directed straight up into the sac, and one would expect the neck plane to be close to perpendicular to the spline as it enters the sac. Choosing the two weighting functions is a non-trivial problem and is discussed in more detail in related work [5].

The cost function that is minimised is

$$
C=\min _{i \in[0, M)} \min _{\phi_{n}, \theta_{n}} \omega_{i}^{m} \omega_{\theta_{n}}^{t} A\left(\phi_{n}, \theta_{n}\right),
$$

where $M$ is the number of images (spline points) in the sequence, and $\mathrm{A}$ is the area of the region of the plane with normal $\left(\phi_{n}, \theta_{n}\right)^{T}$ that is within the vessel lumen. A plot of the minimum plane areas for images in the sequence for a posterior communicating aneurysm is shown in Fig. 2(b). The minimum of the image sequence occurs at image 46, and is shown by the crossed lines.

\section{Labelling the sac}

There is one step remaining before we can compute the working projection angles, and that is to identify the 3D segmentation voxels that belong to the aneurysm sac. We need to do this

\footnotetext{
${ }^{1}$ Note that this does not cause a problem in mislabelling the neck for bilobal sacs, as the neck is always much smaller than the waist-line of the bilobal sac.
} 
because one of our criteria for determining the optimal working projections is to minimise the vessel and sac overlap, so the two structures must be distinctly defined. A feature of spherical projection images computed from points inside the sac is the discontinuity between the lengths of vectors pointing out through the neck, which are large, and the lengths of vectors pointing to the sac walls, which are small [4]. We use this characteristic for labelling the sac.

We use the discrete version of the graduated non-convexity algorithm [9], a method for smoothing an image which allows the smoothing function to break at discontinuities, to identify and exclude the regions of high intensities in a spherical projection image (corresponding to vectors pointing out through the neck) and then interpolate between the neighbouring low intensity regions. Using the interpolated, or 'smoothed' image we can label all voxels that lie within the 'smoothed' distances of the centre voxel (that was used to compute the spherical projection image) as being part of the aneurysm sac.

More specifically, we firstly find the pixels in a spherical projection image $P$ that correspond to the long vectors, pointing out through the aneurysm neck. These pixels are the ones that differ significantly from the rest of the image, based on an estimate of the noise, $\sigma$, in the image. We can estimate the noise in $P$ by comparing $P$ to an image that is a lightly smoothed version of $P$, where breaks in the smoothing function are allowed. Then by creating a heavily smoothed version of $P, S$, where breaks are not allowed, the high intensities in $P$ can be labelled as those pixels that deviate more that $3 \sigma$ from $S$. Finally, $P$ is smoothed, not allowing for breaks, with the exclusion of the labelled high intensity pixels. To label the aneurysm sac we simply identify all voxels along each of the vectors around the sphere that are within the distance specified in the final smoothed image.

We perform this entire process for every spherical projection image from the end of the spline (within the sac) to the image corresponding to the point a quarter of the way along the spline towards the aneurysm neck. We use more than one point (and therefore image) for the labelling process, so as to ensure complete labelling of all lobes of the sac. Often some of the larger aneurysms can be distinctly bilobal. An example of a spherical projection image and its 'smoothed' version, for a middle cerebral artery aneurysm, are shown in Fig. 3(a) and (b). A rendered picture of the final segmented aneurysm sac is shown in Fig. 3(c).
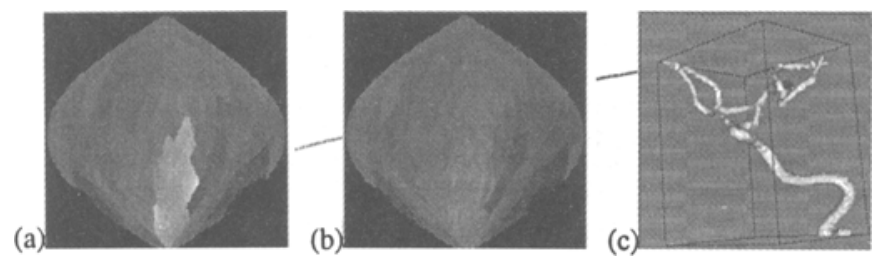

Fig. 3. A spherical projection image (a) computed from a point within the sac of a middle cerebral artery aneurysm, next to $(b)$ the 'smoothed' version of the image. The funal segmentation of the aneurysm is shown in (c). Note that the spherical projection images have been corrected for the distortion caused when spherical coordinates are mapped onto a rectangular grid.

\section{Predicting the working projection angles}

We now have all of the information required to find the optimal working projections. We have based our method for predicting the working projection angles on the same criteria that radiologists use for manual predictions. We have divided the six most common intracranial 
saccular aneurysm sites into two groups, based on the aneurysm geometry. Within each group we quantitatively optimise features of the $\mathrm{X}$-ray projections that are particular to that group. The two groups are illustrated in Fig. 4.
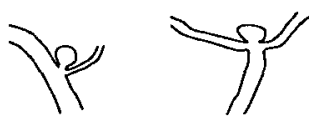

Group A

The group $A$ sites are side-wall aneurysms that arise adjacent to the origin of a vessel that is relatively small with respect to the parent artery. Included in this group are posterior communicating and carotid ophthalmic artery aneurysms. The second set of sites, group $B$, consists of termination aneurysms, where the parent artery divides into two or more branches. Basilar termination, terminal in-

Fig. 4. Aneurysm types. ternal carotid, middle cerebral and anterior communicating artery aneurysms are included in group B. The anatomy at the anterior communicating artery level is complicated, with $80 \%$ of aneurysms occuring at this site being associated with hypoplasia of one of the proximal cerebral arteries (A1) [10]. Hence most aneurysms at this site occur at the junction of the hyperplastic $\mathrm{A} 1$, the $\mathrm{A} 2$ and the anterior communicator, and we therefore class them as being similar to the other bifurcation aneurysms in group $\mathrm{B}$.

The projection criteria for group A are (1) to minimise the overlap of the vessels and the sac in the X-ray image, and (2) to minimise the foreshortening of the parent artery. For group $B$ the criteria are (1) to minimise the overlap of the sac by the left T-junction branch, and (2) to minimise overlap by the right branch. These two optimisations for group B result in two working projections.

Overlap of the sac is measured by fixing the gantry angle and computing the average proportion of $\mathrm{X}$-rays that are attenuated by the sac, compared to the attenuation by the surrounding arteries. If the average normalised proportion is $\alpha$, then values of $1-\alpha$ close to 0 represent little sac overlap, and values closer to 1 indicate more overlap of the sac by arteries.

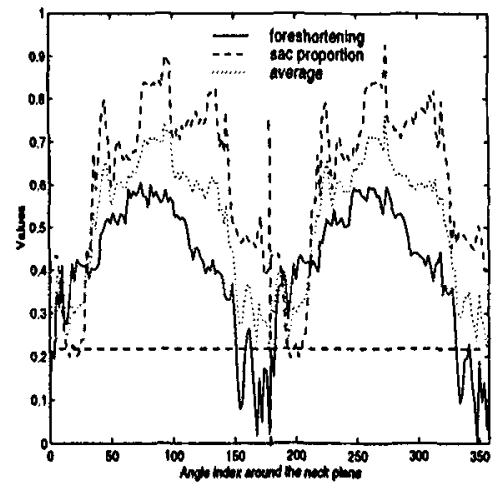

Fig. 5. Plot of the sac overlap and parent artery foreshortening measures, along with their average for the neck plane angles for an anterior communicating artery aneurysm.

Parent artery foreshortening is measured by analysing the section of the parent artery immediately below the aneurysm neck. This arterial section is separated using a method described in previous work [11]. The quantitative value of interest here is the average attenuation of the $\mathrm{X}$-rays passing through the parent artery. When normalised, the average value is minimised at the gantry angle for the $\mathrm{X}$-ray image showing the least foreshortening of the parent artery.

Thus, the optimisation procedure for the aneurysm sites in group $A$ is to take the average between the normalised foreshortening measure and the normalised sac overlap measure, and search for the minimum. We firstly find the minimum over all the gantry angles parallel to the neck plane (see Section 3). Then from this initial angle we do a brute force search through the neighbouring angles, updating the current angle if a better projection under the described criteria is found. If none of the neighbouring angles, up to a radius of 20 degrees, is found to produce a better projection then the search is stopped. Note that we do not consider any angles outside the limits of the X-ray machine gantry. The entire (non-optimised) minimisation procedure takes less than six minutes on a Silicon Graphics $200 \mathrm{MHz}$ workstation. 
The sac overlap and foreshortening measures, along with their average for all angles parallel to the neck plane for an anterior communicating artery aneurysm are shown in Fig. 5 . The minimum is marked with the crossed lines. In the plot the measures are almost, but not quite symmetric at $180^{\circ}$ intervals. This asymmetry is because the beam is cone shaped and not parallel.

The optimisation for the group B sites follows a very similar procedure to that of the group A sites, except that two final angles are found, one for the minimisation of sac overlap from the left, and one for overlap from the right. $\mathrm{X}$-ray images for some of the final prediction angles for a posterior communi-
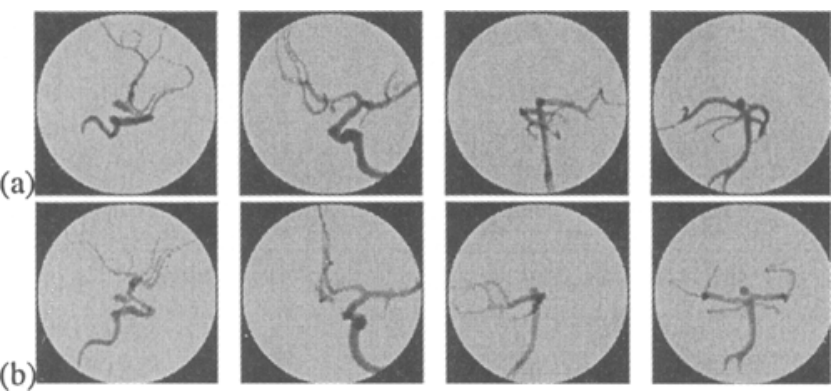

Fig. 6. Left to right in (a) are some of the final prediction results for a cating and an anterior com- posterior communicating and an anterior communicating artery aneurmunicating artery aneur- ysm (both pathological cases), and a synthetically constructed basilar ysm (pathological exam- termination artery aneurysm. The standard views used for angiographic ples), and a synthetically diagnosis screening of the same aneurysm sites are shown in (b). The composed basilar termi- gantry angles for all of these images are given in the table of Fig. 7. nation artery aneurysm are shown in Fig. 6(a). The standard projections for the diagnosis (not coiling) of these three aneurysm sites are shown in Fig. 6(b). The angles for each of the coiling prediction angles and the standard diagnosis angles are given in the table of Fig. 7.

Although the standard diagnosis projections are rarely used as working projections, they are often used as starting points in the manual search for a good working projection. We use them as comparisons to our predicted working projections because it would currently be invalid to compare our results with the actual working projections used during the coilings, as we have not performed a registration between the 3D MRA data and the 2D data acquired during coiling. We are currently carrying out experiments on a method for computing the change in orientation of the patient's head between their position in the MR screening and their position during coiling. When we have this registration information along with the working projection information we will be able to correctly assess our results.

\section{Conclusions}

We have outlined a pre-treatment algorithm for determining the best $\mathrm{X}$-ray gantry angle for viewing intracranial saccular aneurysms during endovascular coiling procedures. There is clearly a necessity for such predictions to reduce the time, radiation and contrast dose

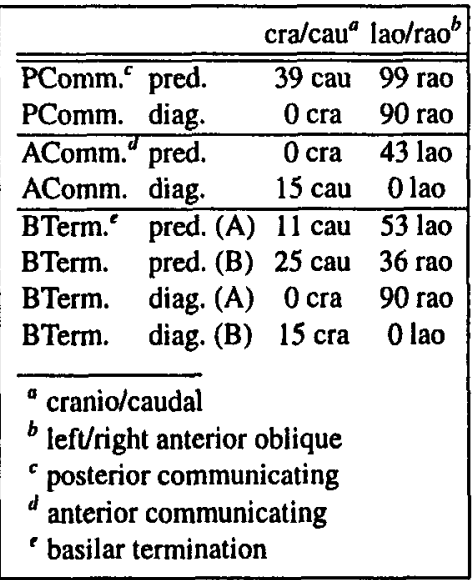

Fig. 7. Gantry angles for the predicted working projections and the standard diagnosis projections. 
associated with GDC treatments, and to provide the radiologist with maximal information regarding the state of the aneurysm.

The results from each step of our optimisation algorithm can be used independently to provide both qualitative and quantitative information to the radiologist prior to coiling. The first step, the segmentation of the vessels, if rendered to show a fully $3 \mathrm{D}$ representation of the vessel structure, can provide important qualitative information about the geometric structure of the arteries and aneurysm. The orientation of the neck, which is often difficult to ascertain by eye, even from 3D data, can be a guide to the radiologist for precise adjustments when inserting the coil. The third step in our algorithm, labelling the sac voxels, can result in an estimate of the aneurysm volume, a measure previously unattainable except by extrapolation from 2D measurements. Finally, the prediction of the working projection angles can avoid repeated injections of contrast agent while the radiologist searches for the best projection, and may provide more information than the projection found by the radiologists' trial and error method.

In conjunction with the work described in this paper we have also developed a planning tool for simulating DSA images, that allows the radiologist to view synthetic X-ray images from any angle prior to coiling. The geometric set-up of the simulations is the same as for the

$\mathrm{X}$-ray machine that is used during coiling. This leaves the finer adjustments on the working projection gantry angle up to the radiologist, who would use our predictions as a guide. Analysis of our predictions in clinical use, incorporating the registration procedure, along with the clinical application of the planning tool will be forthcoming [12,5].

Acknowledgements Thanks are given to Paul Hayton who provided code for the graduated nonconvexity algorithm. DW is supported by the Assoc. Comm. Universities and the AFUW-QLD.

\section{References}

1. G. Guglielmi, F. Vinuela, I. Septka, and V. Macellar. Electrothrombosis of saccular aneuryms via endovascular approach. Part 1. J. Neurosurg., 75:4-7, 1991.

2. J.V. Byrne. Interventional neuroradiology: an emerging subspeciality. Clinical Radiology, 52(12):891-902, 1997.

3. T.W. Malisch, G. Guglielmi, and F. Vinuela et al. Intracranial aneurysms treated with the Guglielmi detachable coil. J. Neurosurg., 87(2):176-183, 1997.

4. R. van der Weide, K.J. Zuigerveld, W.P.Th.M. Mali, and M.A. Viergever. Calculating optimal angiographic angles of cerebral aneurysms. In H. U. Lemke, M. W. Vannier, and K. Inamura, editors, Comp. Assist. Radiol., pages 289-294. Elsevier Science, Amsterdam, June 1997.

5. D.L. Wilson. Planning For Endovascular Treatments of Cerebral Aneurysms. DPhil. thesis, University of Oxford. In preparation, 1998.

6. D.L. Wilson and J.A. Noble. Segmentation of cerebral vessels and aneurysms from MR angiography data. In Proc. IPMI'97, pages 423-428, Poultney, June 1997.

7. C.J. Pudney. Distance-ordered homotopic thinning: A skeletonization algorithm for 3D digital images. Int. J. of Comp. Vis. To appear, 1998.

8. B.A. Barsky. Computer graphics and geometric modeling using beta-splines. Springer-Verlag, London, 1988.

9. A. Blake and A. Zisserman. Visual Reconstruction. MIT Press, Cambridge, Mass, 1987.

10. M.G. Yasargil. Microneurosurgery. Vol Il: Clinical Considerations, Surgery of The Intracranial Aneurysms and Results. Georg Thieme Verlag, Stuttgart, 1984.

11. D.L. Wilson, J.A. Noble, and C.J. Pudney. From MR angiography to X-ray angiography. In Proc. of Med. Im. Understanding and Anal. '97, pages 161-164, Oxford, U.K., July 1997.

12. D.D. Royston. Endovascular Treatment of Intracranial Aneurysms - Preoperative Evaluation and Treatment Planning Using Magnetic Resonance Angiography. MSc. thesis, University of Oxford., 1998. 